\title{
Does COVID-19 restrictions affect the detection and management of ectopic pregnancies?
}

\author{
Melissa Deniz ${ }^{1}$ and Johnson Amu ${ }^{2}$ \\ ${ }^{1}$ Guy's and St Thomas' NHS Foundation Trust \\ ${ }^{2}$ Blackpool Victoria Hospital
}

November 30, 2020

\begin{abstract}
Objective: To assess impact of COVID-19 on diagnosis and management of ectopic pregnancy.Design: Retrospective comparative analysis of ectopic pregnancies (EP) between January and July in 2019 (pre-COVID) and 2020.Setting: Performed at Blackpool Victoria Hospital, a district general hospital in Lancashire. There were no changes in health care personnel and EP management guidelines during both periods, however service provision was modified in line with COVID-19 regulations.Population: We identified $27 \mathrm{EP}$ cases of 1780 total pregnancies presented to early pregnancy unit (EPU) in 2019 compared to 22 cases of 1782 pregnancies in 2020. Methods: Data was collected from EPU computerised database, looking at maternal age, site, side, size and gestation at diagnosis, ruptured EP, different management options. A Chi square statistical analysis compared the variables.Main outcome measures: Gestation at diagnosis and cases of ruptured EP. Secondary outcome measures were the different management options.Results: The incidence of EP cases was similar in 2020 and 2019 (22/1782; 1.23\% Vs 27/1780; $1.51 \%, \mathrm{P}=0.512)$. We found more cases of ruptured EP in 2020 compared to 2019 nonetheless statistically insignificant $(6 / 22$; $27.3 \%$ Vs $5 / 27 ; 18.5 \%, \mathrm{P}=0.467)$. However, a stark difference noted was the gestation of EP diagnosis, $77.3 \%$ presented late ( $>6$ wks amenorrhoea) in 2020 compared to 2019 , proving to be statistically significant $(17 / 22 ; 77.3 \% \mathrm{Vs} 25 / 27 ; 92.6 \%$, $\mathrm{P}<0.001)$. Other parameters like maternal age, site and size of EP, and different management options; were not statistically significant.Conclusions: We urge women to seek help as there are infection prevention measures in place, to provide the services required in early pregnancy.
\end{abstract}

\section{Hosted file}

ectopic covid corrected submit.pdf available at https://authorea.com/users/379911/articles/ 496109-does-covid-19-restrictions-affect-the-detection-and-management-of-ectopicpregnancies 